\title{
Precariedad laboral: concepto, variables y propuesta de análisis*
}

\author{
Precarious work: concept, variables and analysis proposal.
}

\author{
Francisco Nicolás Favieri**
}

\section{Resumen}

El siguiente trabajo refleja las principales discusiones respecto de la precariedad laboral y sus estrategias de medición. Frente a la complejidad del fenómeno, se propone, sobre la base de un ejemplo, la aplicación de un Análisis de Componentes Principales (ACP) que permite, a partir de un conjunto de variables dado, encontrar cuáles tienen mayor peso explicativo en relación con un fenómeno latente, en este caso la precariedad. Para ello, a modo de ejemplo, se trabaja con microdatos de la Encuesta Permanente de Hogares (EPHINDEC) del segundo trimestre de 2015 para el aglomerado Gran San Juan, señalando los pasos y principales hallazgos encontrados en la utilización de esta estrategia.

\section{Abstract}

The following work represents the main discussions about labor precariousness and their metric strategies used. In view of the complexity of the phenomenon, it proposes with an example, the application of the Principal Component Analysis (PCA) which provides information about the participation for each variable in relation with the precariety. Under this approach, the microdata base of Encuesta Permanente de Hogares (EPH-INDEC) form $2^{\text {nd }}$

\footnotetext{
* Artículo recibido 30 de julio de 2016. Aceptado 21 de febrero de 2017.

** Becario Interno Doctoral CONICET, Instituto de Investigaciones Socio Económicas de la Universidad Nacional de San Juan. Correo electrónico: franfavieri@gmail.com
} 
trimester of 2015 in Gran San Juan was investigated (like an example excersie), indicating the steps and main findings founded in the application.

\section{Palabras clave}

Precariedad laboral - Análisis de Componentes Principales (ACP)

- Encuesta Permanente de Hogares (EPH).

\section{Keywords}

Precarious work - Principal Component Analysis (PCA) -

Encuesta Permanente de Hogares (EPH).

\section{Introducción}

A lo largo de la década del setenta se asistió a una trasformación general en el modo de producción imperante. Las políticas de pleno empleo y proteccionismo macroeconómico resultaron agotadas frente a los nuevos objetivos del gran capital internacional (Antunes, 2005; Neffa, 2008; Barattini, 2009). Se aplicaron nuevas estrategias y tecnologías de producción, como también de explotación, control y gestión de la fuerza de trabajo, dando lugar a una reestructuración productiva que transformó las relaciones adoptadas entre el capital y el trabajo.

Estos cambios dieron inicio a un proceso de heterogeneización en la producción generando importantes desequilibrios económicos y profundizando la desigualdad entre sectores, regiones y países (Julian Vejar, 2013). Al mismo tiempo, se asistió a una "metamorfosis" de las formas del trabajo. Se crearon figuras más flexibles y adaptables a los intereses del capital, debilitando al empleo como el principal mecanismo de integración y cohesión social (Gorz, 1995).

Las estrategias de flexibilización ${ }^{1}$ sobre la producción y el trabajo pudieron viabilizar este proceso. Se establecieron nuevas formas de contratación -contratos a plazo fijo, eventuales, temporales- que impuso la "externalización" y subcontratación del trabajo flexibilidad externa-, transfiriendo riesgos empresariales a los trabajadores/as. A la vez que se profundizaron cambios en la jornada de trabajo sobre el ritmo e intensidad laboral -flexibilidad externa-, "polivalencia laboral, salario variable, trabajo en equipo, entre otras" $^{2}$ (Neffa, 2008; Todaro Cavallero, 2016).

\footnotetext{
${ }^{1}$ En principio, este término se diseña instrumentalmente como una teoría posfordista (neoshumpeteriana, regulacionista y de especialización), "como una nueva modalidad de movilización de la fuerza de trabajo vinculada a las innovaciones toyotistas de la organización del trabajo" (Menéndez, 2010:120).

${ }^{2}$ Neffa (2008) y Standing (2011) hablan también de flexibilidad salarial -ajuste de precio del salario según demanda de trabajo-, flexibilidad de número -contratar y despedir sin mayores costos-, flexibilidad funcional -control y gestión de trabajo sin obstáculos internos-, flexibilidad como capacidad de dirección
} 
Durante los noventa, en la Argentina se aplican las políticas de flexibilización laboral conjuntamente con otra serie de reformas de carácter económico como privatizaciones, apertura comercial, suspensión de regímenes de promoción industrial, entre otras, que lograron una fuerte expulsión de mano de obra y un aumento de la precariedad laboral. El empleo formal y típico -asalariado, estable y protegido-fue reemplazado por situaciones de trabajo con mayores niveles de incertidumbre e inseguridad laboral (Henry, 2013; Neffa y De la Garza Toledo, 2010).

En este periodo, la tasa de empleo se contrajo acompañando los ciclos económicos de crisis y de recuperación nacionales (OIT, 2003), mientras que la tasa de desempleo exhibió una tendencia creciente -con picos del 20\% entre 1995 y 2002- debido a la incapacidad de la economía de generar empleos en un contexto de aumento de la tasa de actividad (MTEySS y OIT, 2012; OIT, 2015).

Esta crisis que se inicia en 1998 y desemboca en el estallido de 2001 implicará un cambio de estrategia gubernamental, que se hará evidente a partir de 2003 con el Dr. Néstor Kirchner.

Varios autores acuerdan (Neffa, 2010; Berasueta y Biaffore, 2010; Fernández Milmanda, 2013; Del Bono y Bulloni, 2013, entre otros) que se configuró un nuevo patrón de crecimiento que es resultado de importantes modificaciones en el contexto económico-productivo y en el marco político-institucional, afectando también y de manera particular a las relaciones de trabajo.

Desde 2003 se evidenció una baja de la tasa de desocupación para el total de aglomerados del país, pasando del 20,4\% -primer trimestre de 2003- (EPH-INDEC, 2004) al 6,6\% -segundo trimestre de 2015- (EPH-INDEC, 2015a) paralelamente; la proporción de asalariados sin descuento jubilatorio disminuye de un 48,9\% -cuarto trimestre de 2004- (EPH-INDEC, 2005) hacia un 33,1\% -segundo trimestre de 2015(EPH-INDEC, 2015b).

En la provincia de San Juan, el sector que más aporta al PBG es el sector servicios, ofreciendo la mayor cantidad de empleos privados registrados $-53 \%$ para el 2014(Observatorio de Empleo y Dinámica Empresarial-MTEySS-SIPA, 2014) con relación al resto de las actividades. Así mismo, las empresas privadas por sector de actividad y tamaño posicionan en un $65 \%$ la concentración sobre las actividades terciarias, siendo las microempresas -61\%- las mayoritarias en el sector (Boletín de Áreas EconómicasÁreas Económicas Locales -2014- ).

En cuanto al mercado de trabajo y sus indicadores tradicionales, la tasa de desocupación disminuye paulatinamente desde primer semestre de 2003 hasta el segundo trimestre de 2015 en un 6,7\%, a la vez que la tasa de empleo se sostiene en

empresarial -la empresa define competencias y habilidades necesarias para el trabajo/trabajador/a(Standing, 2011 en Cuevas Valenzuela, 2015). 
valores cercanos al 35-36\%, con periodos de crecimiento (2003-2008) y descenso (2009-2014) con variaciones positivas en el cuarto trimestre de 2008 y 2011.

La tasa de subocupación horaria demandante conserva una tendencia descendente entre 2003 y 2013, excepto a partir del tercer trimestre de 2013 hasta el segundo trimestre de 2015 (5,1\%), cuando sus valores aumentan (EPH-INDEC, 2015).

En este caso, las tasas expresan un comportamiento similar al observado a nivel nacional. El crecimiento de la tasa de ocupación se ralentiza después de la crisis 20082009, a la vez que la subocupación aumenta levemente.

Por otra parte, los asalariados sin descuento jubilatorio para el segundo trimestre de 2015 representan el 41,5\%, evidenciando una fuerte presencia de empleos en situación de precariedad (EPH-INDEC, 2015b).

Estos indicadores muestran -a nivel general- la persistencia del fenómeno de la precariedad; sin embargo, resulta pertinente determinar cuáles variables tienen más influencia y participación en la conformación de situaciones de precariedad, en este caso, San Juan ${ }^{3}$. Para ello, y este es el motivo del artículo, se propone la aplicación de una técnica de análisis multivariado denominado "análisis de componentes principales" (ACP) que indicará, en un grupo dado de variables relacionadas de forma latente con el fenómeno de la precariedad, cuál/es tiene/n mayor peso relativo $\mathrm{y}$, por tanto, encontrará alguna explicación que permita comprender más acabadamente este fenómeno.

Si bien la aplicación de este análisis es de tipo exploratorio, ya que no determina concluyentemente las causas y/o motivos de las situaciones de precariedad, y puede ofrecer recursos de interés para investigaciones más específicas.

Este artículo se divide en cuatro partes. Primero, se esbozan algunas reflexiones en torno a la informalidad y precariedad laboral: definiciones y medición; posteriormente el apartado metodológico destaca paso a paso la aplicación del análisis de componentes principales (ACP) dando cuenta de la pertinencia de las variables utilizadas y su operacionalización. La tercera parte consiste en el análisis de los resultados y, por último, las conclusiones.

\section{Informalidad y precariedad laboral: definiciones y medición}

El empleo asalariado fue el principal protagonista-modelo después de la segunda posguerra. Como depositario parcialmente exitoso de los históricos reclamos de la clase obrera, los códigos y la legislación laboral de la época perfilaban un empleo bajo contrato por tiempo indeterminado, estable, debidamente registrado ante la ley -con

\footnotetext{
${ }^{3}$ La aplicación de ACP sobre el aglomerado Gran San Juan se atribuye a que los elementos presentados en este artículo forman parte de una investigación mayor -tesis doctoral- que estudia la relación entre la juventud trabajadora del comercio y su organización gremial en un contexto de crisis económica y precarización del empleo. Estudios similares se han realizado para el total de aglomerados de Argentina (Fernández Massi, 2014; Pol, Guilló y Maradona, 2015), encontrando resultados similares.
} 
beneficios como jubilación, aguinaldo, vacaciones pagas- y cuyas jornadas no excedían las 8 horas, y en caso de hacerlo, el pago de horas extras era obligatorio. El lugar de trabajo se encontraba fuera del ámbito doméstico y era corriente desempeñarse sólo en una actividad. Existía una clara relación de dependencia y subordinación a un solo empleador (Neffa, 2008). Esto se define como "empleo típico" y aún hoy existe, aunque sólo para un número cada vez más reducido de trabajadores/as.

Los empleos resultantes de la aplicación de modelos flexibilizadores sobre la relación de trabajo permitieron la aparición de empleos cuya principal modificación sobre el contrato de trabajo es el tiempo de finalización: pueden existir contratos por algunas horas, por temporada, por alguna eventualidad; en lugares de trabajo diferentes por vez o en el hogar, con actividades también cambiantes e incluso simultáneas, salarios ajustados por objetivos o productividad. La relación con el empleador puede ser a través de un tercero -tercerización- y éste a la vez puede establecer subcontratos. Estas formas "atípicas", a pesar de ser legales, se complejizan si estas posibles formas de contrato no están registradas. Los principios "protectorios" y de seguridad social que aún persisten en la legislación de trabajo dejan de acompañar a los/as trabajadores/as desmejorando su situación respecto de aquellos/as que sí gozan de estos derechos.

Existen también figuras intermedias-precarias -frecuentemente ilegales-, donde podría hablarse de registro "deficiente" o "solapamiento". Figuras donde, por ejemplo, un empleado trabaja cumpliendo horarios y directivas -relación de dependencia-, pero factura como vendedor de servicios -por tarea terminada, cuenta propia-, o se declara la mitad del sueldo real devengado y el resto es cobrado "en negro", a ello pueden sumarse inconvenientes en recibos de sueldo, no respeto de días libres, horarios, horas extra, entre otros.

Estas formas de trabajo son las que se denominarán como precarias, haciendo énfasis en "la falta", "la inestabilidad", "la inseguridad". Los primeros estudios al respecto los asignaron como formas atípicas de empleo, relacionadas directamente con las "economías informales" 4 .

Desde los años cincuenta se había señalado la existencia de trabajos "marginales" que fueron conceptuados como una característica de los países en vías de desarrollo (Lewis, 1958 en Neffa, 2008) o, en todo caso, población excedente, marginal, de reserva para los teóricos de la dependencia (Quijano, 1971; Nun, Marín y Murmis, 1968; Nun, 1969; Cardoso, 1971; De Oliveira, 1973 en Neffa, 2008) y estos trabajos "disfuncionales" eran propios de la periferia mundial.

OIT, recuperando algunas de estas tradiciones, desarrolla en 1972 el concepto de "economía informal", cuya expresión se define en trabajos no institucionalizados, no

\footnotetext{
${ }^{4}$ Los trabajos, según el modelo típico, se terminan instituyendo como un "ideal" estructural del modelo fordista. Sólo en algunos países centrales del occidente capitalista esta forma de trabajo se abrió camino. Para el resto, como en América Latina o la Argentina, estos empleos típicos se circunscribían en algunos sectores de la actividad económica, fundamentalmente urbanos.
} 
estructurados, cuyos objetivos se dirigen a "maximizar el ingreso total familiar para asegurar la sobrevivencia" (Neffa, 2008: 4) de quienes se encontraban en esa situación, eran frecuentemente trabajadores pobres -excedentes- que no podían emplearse en las modernas empresas de las zonas urbanas. Entonces, según Neffa (2008), se naturaliza bajo esos conceptos la denominación de "sector informal urbano" y se replica hacia toda Latinoamérica (Pok, 1992; De la Garza, 2009).

Es así que desde sus indicadores terminan definiendo al sector informal en la existencia de unidades de pequeña escala, de organización rudimentaria, sin mucha distinción entre capital y trabajo, cuyas relaciones de empleo son ocasionales, de parentesco, frecuentemente personales, donde no existen garantías ni registros -de seguridad social o salarial. Terminan integrando este ámbito cuentapropistas excluyendo profesiones liberales-, microempresas de hasta 5 ocupados, trabajadores familiares no remunerados y personal doméstico (Neffa, 2008).

La informalidad, como fenómeno, se acrecentó frente a la revolución iniciada en los setenta (Neffa, 2008; Barattini, 2009), y sus límites conceptuales también comenzaron a desdibujarse, en particular, sobre los aspectos relativos al sector de actividad económica, tamaño del establecimiento productivo y las lógicas de contratación5.

Las nuevas formas de empleo trascendían la ilegalidad, se posicionaban en los espacios reservados conceptualmente para los empleos típicos, pero en muchos casos sus características eran similares a las de los empleos informales (Pok, 1992; De la Garza, 2009). El fenómeno advertido como poco corriente, poco a poco comenzó a tomar protagonismo en el contexto de una creciente heterogeneización productiva.

En los años ochenta, la OIT delimita el concepto de "precario" asociando algunas dimensiones de las llamadas "condiciones y medio ambiente de trabajo" (CYMAT) en relación con la inestabilidad y carencia de salario indirecto (Orsatti, 1989 en Menéndez, 2010).

Uno de los primeros autores en conceptualizar la precariedad es Guy Caire (1982), quien sostiene que la naturaleza del contrato y la "identificación" del empleador son dimensiones claves para determinar la existencia de la precariedad. Con esto se refiere a las formas que el empleo adquiere según el tipo de contrato -duración determinada, en negro, eventual, temporal, subcontrato, part time, a domicilio, etc.

Otros trabajos insignia, como los de Córdova (1985), Galin y Feldman (1990) y Rodgers (1992), ponen énfasis en la duración del contrato como una de las principales características de un empleo precario, asociando la noción de incertidumbre en tanto estabilidad e inseguridad en relación con las situaciones de empleo que no se encuentran debidamente registradas $y$, por tanto, pone en riesgo el futuro de ese/a

\footnotetext{
${ }^{5}$ Pueden existir empleos "fuera de la economía informal" en establecimientos de menos de 5 personas, debidamente registrados, con contratos eventuales o temporarios -flexibles- con jornadas y actividades variables, entre otras. Pueden ser empresas de servicios editoriales, gráficas, de desarrollo de software, etc. (Antunes y Braga, 2009).
} 
trabajador/a6. Neffa (2008), por ejemplo, señala este punto como un importante elemento diferenciador de una situación de precariedad, en consonancia con autores/as como Pok (1992), Mora y Oliveira (2009 y 2010) y Escoto (2013).

Pero entre la legalidad y la ilegalidad, lo formal y lo informal, las fragmentarias figuras de empleo que emergen en este fenómeno hacen a la existencia de un "continuum o gradualización" (Ferrari y López en Medina, 2008) de relaciones precarias de trabajo donde algunos elementos adquieren un protagonismo diferencial, de acuerdo con Beccaria y Serino (2001) y Suárez et al (2005) en relación a la existencia de "estabilidad"; Lindenboim (2000 y 2003); Fernández Massi (2014); Pol, Guilló y Maradona (2015) destacando comportamientos específicos de los empleos precarios según el sector de actividad económica en el que se desarrollan. De la Garza (2010), Castel (2010), Dasten (2013), Antunes (2003 y 2005), Longo (2012) también señalan la importancia de las distintas figuras del empleo precario, sus yuxtaposiciones en la economía y su relación directa con las transformaciones en la estructura productiva y la regulación, incluyendo además los debates en relación a los sindicatos.

Por otra parte, a los elementos básicos de análisis -horas, salario, lugar de trabajo, sector de actividad y actividades- agregan aspectos relativos a lo "subjetivo" y las transformaciones en la experiencia de los cuerpos y las emociones (Paugman, 2000; Boufartigue, 2007; Barattini, 2009; Piñeiro, 2013) señalando la aparición de hiperactividad, simultaneidad, intimidades fluidas, inestabilidad, agotamiento afectivo, etc. (Barattini, 2009).

Esta variedad y riqueza conceptual y analítica hace que no exista una "única" definición de "precariedad", sino una serie de variables que ayuda a determinar situaciones más cercanas o lejanas a una relación laboral precaria. En este sentido, se habla de "precariedad multidimensional" advirtiendo que las situaciones de inestabilidad, inseguridad e insuficiencia se articulan sobre las jornadas de trabajo, las actividades, el salario, el lugar de trabajo y su impacto sobre las subjetividad de los/as trabajadores/as. Utilizan términos similares y trabajan sobre esta idea Guadarrama Olivera, Hualde Alfaro y López Estrada (2012), Mora Salas (2005), Menéndez (2010), Julian Vejar (2013), Fernández Massi (2014), Pol, Guilló y Maradona (2015), entre otros/as.

\section{Metodología: medición multidimensional de la precariedad}

Dado que la precariedad como tal ha sido definida como un fenómeno multidimensional, se quiere saber qué grupo de variables ayuda a explicarla mejor para posteriores estudios. Para ello, y en vinculación con los trabajos desarrollados por Fernández Massi (2014) y Pol, Guilló y Maradona (2015) sobre estudios de precariedad,

\footnotetext{
${ }^{6}$ Para García y De Oliveira (2012), la noción de precariedad en este punto busca complementar nociones como la de subempleo o informalidad.
} 
se aplicará a la base individual de la Encuesta Permanente de Hogares -aglomerado Gran San Juan, Argentina- el Análisis de Componentes Principales (ACP).

Este análisis permite la creación de subgrupos de variables -espacios factoriales- que independientes entre sí están relacionadas en torno al fenómeno latente que se quiere encontrar, siendo cada componente el resultante de un grupo de variables que prevalecen sobre otras según su capacidad explicativa y en función de la variabilidad que representan al interior de la muestra. Esto permite a la vez una lectura entre los distintos componentes, permitiendo determinar cuáles grupos de variables del conjunto tienen más poder explicativo sobre alguna situación de precariedad.

En principio, cada variable de trabajo es independiente, sin embargo, aquí se reúnen bajo los criterios teóricos fundamentados en la definición multidimensional de la precariedad, esto es, aspectos que tienen que ver con los derechos laborales, la forma de contratación, jornada de trabajo y el salario, etc.

Es necesario destacar que la aplicación de estos métodos multivariados, con algunas variaciones en tanto selección de variables, son aplicados regularmente en los estudios sobre mercado de trabajo, como se exponen en las obras de Mora (2006 y 2009), García Guzmán (2008 y 2009), García y de Oliveira (2012) y Escoto (2013).

Se utilizará, además, el software de análisis estadístico SPSS e InfoStat para el tratamiento de datos.

\section{Preparación de la base de datos}

A partir de la base de microdatos de la EPH del segundo trimestre, se seleccionan aquellos casos que cumplan con la condición de asalariado ocupado, excluido el servicio doméstico. Se seleccionan las variables relacionadas al concepto multidimensional de precariedad y se recodifica de tal forma que exprese un orden de sentido (Fernández Massi, 2014), en tanto que valores iguales a 1 expresen "mayor precariedad" y los iguales a " 0 " "menor precariedad" 7 .

Debido a que todas las variables seleccionadas para el estudio no son dicotómicas y tienen un nivel de medición diferente, se determinó lo siguiente:

- Antigüedad: para trabajos cuya duración continua es declarada con menos de un año, se recodifica con "1" ("mayor precariedad"). A quienes declararon tener una antigüedad mayor a un año, se les asigna "0" ("menor precariedad") 8 .

- Turno: para los turnos diurnos y nocturnos, se asigna "0" ("menor precariedad"); para los rotativos, "1" ("mayor precariedad").

- Ingresos: integrada por Monto de ingreso de la ocupación principal percibido en el mes de referencia (Salario), menos (-) Salario Mínimo, Vital y Móvil (SMVM) y

\footnotetext{
${ }^{7}$ De igual forma, Castillo Fernández (2008) incluye las variables dicotomizadas en su análisis sobre la precariedad en Panamá, al utilizar el análisis de componentes principales (ACP).

${ }^{8}$ Se infiere que a mayor antigüedad en el trabajo existe mayor estabilidad, aspecto de "no precariedad"
} 
Deciles de ingreso de la ocupación principal del TOTAL EPH. En ambas variables, aquellos valores de ingreso que superen los $\$ 4.176$ (Salario Mínimo, Vital y Móvil para el segundo trimestre de 2015) se asignará "0" y a los que estén por debajo del SMVM se les asignará "1".

De esta manera, se unifican los niveles de medición asegurando un desempeño acorde con los estadísticos a aplicar en el Análisis de Componentes Principales.

Cuadro $\mathbf{N}^{\circ}$ 1: Descripción de datos, presentación de variables y condición de orden.

\begin{tabular}{|c|c|}
\hline Unidad de análisis & $\begin{array}{l}\text { Individuos ocupados asalariados (obreros u empleados), excluido } \\
\text { servicio doméstico del aglomerado Gran San Juan. }\end{array}$ \\
\hline N Muestral & 477 \\
\hline N Ponderado & 128154 \\
\hline Variables & ión de orden \\
\hline $\begin{array}{l}\text { Deciles de ingreso de } \\
\text { la ocupación principal } \\
\text { del TOTAL EPH }\end{array}$ & $\begin{array}{l}\text { Salarios situados en los deciles que no superan los } \$ 4176 \text {, mayor } \\
\text { precariedad y se recodifican con " } 1 \text { ". Salarios cercanos a los deciles } \\
\text { más altos que superan los } \$ 4176 \text {, se recodifica con " } 0 \text { ", menor } \\
\text { precariedad. }\end{array}$ \\
\hline 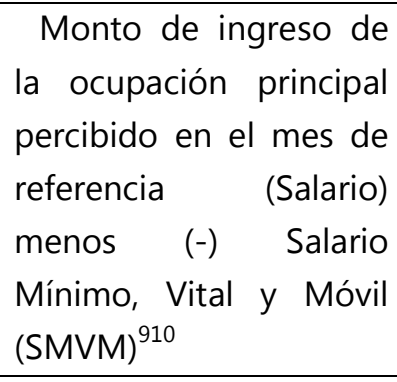 & $\begin{array}{l}\text { Los valores obtenidos de la diferencia entre Monto de ingreso de } \\
\text { la ocupación principal percibido en el mes de referencia y el Salario } \\
\text { mínimo, vital y móvil (SMVM) son comparados nuevamente con el } \\
\text { SMVM. Aquellos que superan } \$ 4176 \text { se les asigna "0" ("menor } \\
\text { precariedad") y los que no lo hacen "1" ("mayor precariedad"). }\end{array}$ \\
\hline Antigüedad & $\begin{array}{l}\text { Recodifica de manera tal que trabajos con antigüedad menor a un } \\
\text { año, "1" (mayor precariedad) y mayores al año, "0" (menor } \\
\text { precariedad). }\end{array}$ \\
\hline $\begin{array}{l}\text { Búsqueda de otro } \\
\text { empleo }\end{array}$ & $\begin{array}{l}\text { Si busca otro empleo, se asigna "1" (mayor precariedad), en caso } \\
\text { contrario, " } 0 \text { " (menor precariedad). }\end{array}$ \\
\hline $\begin{array}{l}\text { Subocupación } \\
\text { demandante }\end{array}$ & $\begin{array}{l}\text { Si cumple con la condición de subocupación demandante (trabaja } \\
\text { menos de } 40 \text { horas semanales y busca activamente trabajo), se le } \\
\text { asigna " } 1 \text { " (mayor precariedad) y " } 0 \text { " en caso contrario. }\end{array}$ \\
\hline
\end{tabular}

9 El SMVM fue de $\$ 4176$ para ese trimestre.

10 El bloque "ingresos" como medición de precariedad tiene relación con el sostenimiento de la unidad familiar y la satisfacción de las necesidades del trabajador, se ve cómo el SMVM es una medida bien correspondida para señalar este aspecto (Fernández Massi, 2014; Pol, Guilló y Maradona, 2015).

11 Se enfatiza como medida de no-precariedad las 8 horas diarias por jornada o las 40 horas semanales de trabajo como la medida "normal" de expresión de un trabajo que permite "el equilibrio adecuado para la salud y la vida social entre la actividad laboral y otras actividades" (Fernández Massi, 2014:241). Si existe sobreocupación, se presume necesidad económica, aun así puede ser una situación impuesta por el empleador, pero en cualquier caso es expresión de precariedad.

12 Con subocupación se indicaría la insuficiencia de esa ocupación para sostener la reproducción simple de la unidad familiar (Fernández Massi, 2014). 


\begin{tabular}{|c|c|}
\hline Sobreocupación & $\begin{array}{l}\text { Si cumple con la condición de sobreocupación (trabaja más de } 40 \\
\text { horas semanales), se le asigna " } 1 \text { " (mayor precariedad) y " } 0 \text { " en caso } \\
\text { contrario. }\end{array}$ \\
\hline Variables & Condición de orden \\
\hline Duración del contrato & $\begin{array}{l}\text { Pueden ser CDI (contratos de duración indeterminados) y expresan } \\
\text { menos incertidumbre y mayor estabilidad, lo que indica } \\
\text { "indirectamente" menor precariedad, mientras que los CDD } \\
\text { (contratos de duración determinados) expresan lo contrario. A los } \\
\text { CDI se les asigna "0" ("menor precariedad" y "1" a los CDD ("mayor } \\
\text { precariedad"). }\end{array}$ \\
\hline Tipo de trabajo & $\begin{array}{l}\text { Plan de empleo, período de prueba y beca/pasantía. En cualquiera } \\
\text { de los tres casos, las respuestas afirmativas indican } \\
\text { inestabilidad/precariedad. Se asigna "1" a respuestas afirmativas } \\
\text { ("mayor precariedad") y "0" a respuestas negativas ("menor } \\
\text { precariedad"). }\end{array}$ \\
\hline Turnos & $\begin{array}{l}\text { Al considerar los turnos habituales de trabajo, se considera que un } \\
\text { turno de día es menos precario que un turno de noche y mucho } \\
\text { más que un turno rotativo. Se asigna a turnos diurnos y nocturnos } \\
\text { "0" ("menor precariedad") y " } 1 \text { " ("mayor precariedad") a turnos } \\
\text { rotativos. }\end{array}$ \\
\hline $\begin{array}{l}\text { Derechos laborales y } \\
\text { afines }\end{array}$ & $\begin{array}{l}\text { Vacaciones pagas, aguinaldo, días pagos por enfermedad, obra } \\
\text { social, descuento jubilatorio y recibo de sueldo. Las respuestas } \\
\text { afirmativas indican "menor precariedad". Se asigna "0". En caso } \\
\text { negativo, se asigna " } 1 \text { " ("mayor precariedad"). }\end{array}$ \\
\hline
\end{tabular}

Fuente: elaboración propia sobre la base de la Encuesta Permanente de Hogares (EPH).

Entre las 15 variables seleccionadas se cuentan 30 datos faltantes que representan el $0,419 \%$ del total en valor muestral (Ver anexo 1 ).

Se aplica el procedimiento de Imputación Múltiple (IM) para incorporar los datos faltantes. En este caso, su utilización es conveniente, dado que la no respuesta es baja y se sugiere emplearlos especialmente cuando se utilizan técnicas de análisis multivariado (Medina y Gavlán, 2007: 35).

Una vez ponderados los datos, se aplica el Análisis de Componentes Principales.

\section{Análisis}

Para determinar estadísticamente si las variables propuestas se relacionan con un mismo fenómeno, en este caso la precariedad -como variable no observable o latente-, se aplica el coeficiente Kaiser-Meyer-Olkin (KMO), el cual expresará la viabilidad en la aplicación del análisis de los componentes, esto es, si pueden compararse y además explicar la variabilidad de la muestra. Este coeficiente que expresa la medida de adecuación muestral puede adoptar valores entre 0 y 1 ; si se acerca a 0 , la variación es explicada por correlaciones parciales -lo cual indicaría la inestabilidad del análisis-, 
mientras que si los valores están cercanos a 1, indica que la variación puede explicarse por correlaciones conjuntas. En este caso, con las 15 variables propuestas, el coeficiente obtenido es de 0,881, indicando una buena adecuación muestral, lo que permite continuar con la aplicación de los estadísticos.

La prueba de esfericidad de Bartlett, por su parte, contrasta la hipótesis nula de que la matriz de correlaciones es una matriz de identidad, en cuyo caso no existirían correlaciones significativas entre las variables y el modelo factorial no sería pertinente. Sin embargo, aquí los resultados muestran que el modelo es el adecuado.

Cuadro $\mathbf{N}^{\circ}$ 2: KMO y prueba de Bartlett

\begin{tabular}{|ll|l|}
\hline \multicolumn{2}{|l|}{ Medida de adecuación muestral de Kaiser-Meyer-Olkin } &, 881 \\
& Chi-cuadrado aproximado & 1666472,993 \\
Prueba de esfericidad de Bartlett & gl & 105 \\
& Sig. &, 000 \\
\hline
\end{tabular}

Fuente: elaboración propia sobre la base de datos de EPH-INDEC.

Una vez comprobada la viabilidad de la técnica, se aplican los estadísticos. El análisis factorial muestra 4 componentes -véase cuadro $N^{\circ} 4$ - que refieren a la variable latente (precariedad), aun así, la representatividad de cada variable en cada espacio factorial no es la misma.

Las comunalidades presentes en el cuadro $\mathrm{N}^{\circ} 3$ indican la porción de la varianza explicada por los factores comunes de una variable. Se observa entonces que las más representativas -las que mejor captaron los componentes extraídos- son las cercanas a 1. Cada comunalidad es igual a la suma de los cuadrados de las cargas factoriales, si los valores se encuentran superando el valor 0,6 , entonces puede decirse que la variable se encuentra bien representada.

Las variables como "cantidad de ocupaciones", "sobreocupación horaria", "antigüedad" y "duración del contrato" tienen mayor correlación con otros ejes no considerados en este modelo, aun así, permanecerán para ser analizadas.

Cuadro No 3: Comunalidades

\begin{tabular}{|l|l|l|}
\hline & Inicial & Extracción \\
\hline Cantidad de ocupaciones & 1,000 &, 158 \\
Búsqueda de otro empleo & 1,000 &, 818 \\
Subocupación horaria demandante & 1,000 &, 735 \\
Sobreocupación horaria & 1,000 &, 471 \\
Antigüedad & 1,000 &, 172 \\
Turno & 1,000 &, 713 \\
Recibo de sueldo & 1,000 &, 827
\end{tabular}




\begin{tabular}{|l|l|l} 
Duración del contrato & 1,000 &, 426 \\
Vacaciones pagas & 1,000 &, 891 \\
Aguinaldo & 1,000 &, 950 \\
Días pagos por enfermedad & 1,000 &, 950 \\
Obra social & 1,000 &, 941 \\
GD_Ingreso ocupación principal & 1,000 &, 645 \\
Ingreso-SMVM & 1,000 &, 700
\end{tabular}

Método de extracción: Análisis de Componentes principales.

Fuente: elaboración propia sobre la base de datos de EPH-INDEC.

Si bien cada componente expresa el conjunto total de variables, existen algunas que saturan más que otras, definiendo el perfil del componente, esto a la vez permite determinar la importancia que tiene cada uno en tanto explicación porcentual de la varianza total de los datos presentados; es así que contando 4 componentes de análisis, el primero explica el 41,58\% de la varianza, obteniendo un gran poder explicativo respecto del resto de los componentes. En conjunto, los 4 expresan el $68,74 \%$ de la varianza total.

Como se observa en el cuadro $N^{\circ} 5$, a medida que se agregan componentes, aumenta el poder explicativo total -varianza total acumulada-; sin embargo, a medida que las saturaciones se dispersan en cada conjunto factorial, el porcentaje de la varianza explicada por componente disminuye.

Cuadro $N^{\circ}$ 4: Varianza total explicada

\begin{tabular}{|c|c|c|c|c|c|c|c|c|c|}
\hline \multirow{2}{*}{ 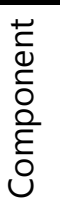 } & \multicolumn{3}{|c|}{ Autovalores iniciales } & \multicolumn{3}{|c|}{$\begin{array}{l}\text { Sumas de las saturaciones al } \\
\text { cuadrado de la extracción }\end{array}$} & \multicolumn{3}{|c|}{$\begin{array}{l}\text { Suma de las saturaciones al } \\
\text { cuadrado de la rotación }\end{array}$} \\
\hline & Total & $\begin{array}{c}\text { \% de la } \\
\text { varianza }\end{array}$ & $\begin{array}{l}\% \\
\text { acumulado }\end{array}$ & Total & \begin{tabular}{|c|}
$\%$ de \\
varianza
\end{tabular} & $\begin{array}{l}\% \\
\text { acumulado }\end{array}$ & Total & \begin{tabular}{|c|}
$\%$ de la \\
varianza
\end{tabular} & $\begin{array}{l}\% \\
\text { acumulado }\end{array}$ \\
\hline 1 & 6,237 & 41,583 & 41,583 & 6,237 & 41,583 & 41,583 & 5,814 & 38,760 & 38,760 \\
\hline 2 & 1,728 & 11,521 & 53,104 & 1,728 & 11,521 & 53,104 & 1,768 & 11,786 & 50,547 \\
\hline 3 & 1,224 & 8,161 & 61,265 & 1,224 & 8,161 & 61,265 & 1,493 & 9,955 & 60,502 \\
\hline 4 & 1,122 & 7,479 & 68,744 & 1,122 & 7,479 & 68,744 & 1,236 & 8,243 & 68,744 \\
\hline 5 & 1,029 & 6,858 & 75,602 & & & & & & \\
\hline 6 & ,946 & 6,307 & 81,910 & & & & & & \\
\hline 7 & ,762 & 5,083 & 86,993 & & & & & & \\
\hline 8 & ,626 & 4,176 & 91,169 & & & & & & \\
\hline 9 &, 535 & 3,564 & 94,732 & & & & & & \\
\hline 10 & ,321 & 2,141 & 96,873 & & & & & & \\
\hline 11 & 220 & 1,468 & 98,341 & & & & & & \\
\hline 12 & , 108 & 717 & 99,057 & & & & & & \\
\hline 13 & 070 & 469 & 99,527 & & & & & & \\
\hline 14 & ,046 & ,309 & 99,835 & & & & & & \\
\hline
\end{tabular}




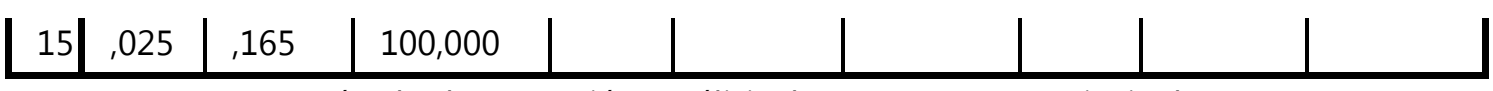

Método de extracción: Análisis de Componentes principales

Fuente: elaboración propia sobre la base de datos de EPH-INDEC.

Ahora bien, entre los componentes de más peso, se seleccionan los primeros 4 -por su representatividad. El cuadro $N^{\circ} 5$ muestra qué variables dan peso a la varianza explicada en cada componente, adquiriendo un perfil explicativo particular según la saturación de las variables que lo integran.

Cuadro No 5: Matriz de componentes rotados ${ }^{a}$

\begin{tabular}{|l|l|l|l|l|}
\hline \multirow{2}{*}{} & \multicolumn{4}{l|}{ Componente } \\
\cline { 2 - 5 } & 1 & 2 & 3 & 4 \\
\hline Descuento jubilatorio &, 967 &, 037 &, 106 &,- 041 \\
Aguinaldo &, 962 &, 073 &, 136 &,- 017 \\
Obra social &, 962 &, 051 &, 108 &,- 019 \\
Días pagos por enfermedad &, 941 &, 051 &, 158 &,- 024 \\
Vacaciones pagas &, 933 &, 047 &, 138 &,- 009 \\
Recibo de sueldo &, 897 &, 068 &, 130 &, 001 \\
Duración del contrato &, 532 &, 346 &, 021 &,- 152 \\
Antigüedad &, 303 &, 206 &, 170 &, 094 \\
Búsqueda de otro empleo &, 125 &, 889 &, 109 &, 003 \\
Subocupación & &, 848 &, 006 &,- 116 \\
demandante &, 047 &, 806 & \\
Ingreso-SMVM &, 137 &, 090 &, 812 &,- 116 \\
GD_Ingreso Ocupación Principal &, 222 &, 042 &, 760 &,- 127 \\
Cantidad de ocupaciones &,- 039 &,- 006 &,- 332 &,- 215 \\
Turno &,- 030 &, 104 &,- 015 &, 837 \\
Sobreocupación horaria &,- 040 &,- 238 &,- 041 &, 641 \\
\hline \% de la varianza & 41,53 & 11,51 & 8,16 & 7,47 \\
\hline
\end{tabular}

Método de extracción: Análisis de componentes principales.

Método de rotación: Normalización Varimax con Kaiser.

a. La rotación ha convergido en 5 iteraciones.

Fuente: elaboración propia sobre la base de datos de EPH-INDEC.

Sobre la base del perfil de saturación de cada componente se definen a los componentes como:

\section{Componente 1: Derechos laborales}

Compuesto por las variables "descuento jubilatorio", "aguinaldo", "obra social", "días pagos por enfermedad", "vacaciones pagas" y "recibo de sueldo", expresan en conjunto algunos atributos que por norma se exigen en la Ley de Contrato de Trabajo (Ley $N^{\circ}$ 20.744). 
Estas variables representan el $41,53 \%$ de la varianza explicada, teniendo un fuerte peso en la definición del fenómeno de precariedad. Tal como señalan Fernández Massi (2014) y Pol, Guilló y Maradona (2015), los estudios dirigidos a analizar situaciones de precariedad suelen valerse de alguno de estos atributos, en especial "descuento jubilatorio" para determinar, a partir de la condición de registro, si ese asalariado se encuentra en la dimensión de precariedad. Los resultados obtenidos de estudios de este tipo explicarían sin problemas parte importante del fenómeno de la precariedad.

\section{Componente 2: Demanda de empleo}

En este componente, cuyo poder explicativo es de sólo 11,52\%, es integrado por las variables de "búsqueda de otro empleo" y "subocupación horaria demandante". Ambas relacionadas frente a la necesidad de un nuevo trabajo. Las causas asociadas a ello pueden interpretarse relacionadas a las remuneraciones, a la estructura del mercado de trabajo, entre otros (Muñoz Izquierdo, 2006; Arango, Escobar y Monsalve, 2013).

\section{Componente 3: Ingresos}

Este componente, que explica el $8,16 \%$ de la varianza, destaca a las variables que tienen como denominador común al ingreso: "Salario de la ocupación principal menos el Salario Mínimo, Vital y Móvil (SMVM)" y los "Deciles de Ingreso de la ocupación principal del total de la EPH". Incluir el aspecto salarial a estudios de precariedad puede complementar y explicar situaciones de precariedad como atributo, en muchos casos definitorio (Merklen, 2004; Acosta Reveles, 2011; Fernández Massi, 2014).

\section{Componente 4: Turnos}

Este componente aporta el 7,47\% de la varianza. La variable con más representación aquí es "turno", señalando la importancia relativa en tanto a la organización en el trabajo como un aspecto de relevancia (Guadarrama Olivera, Hualde Alfaro y López Estrada, 2012).

A continuación, se presenta un biplot donde se incluyen sólo componentes 1 y 2 -que explican en conjunto la mayor variabilidad- para observar, sobre el eje del componente con más representatividad -CP1 "Derechos laborales"-, su relación con el resto de las variables. 
Figura $\mathbf{N}^{\circ}$ 1: Biplot. Distribución de variables de análisis según componentes 1 y 2.

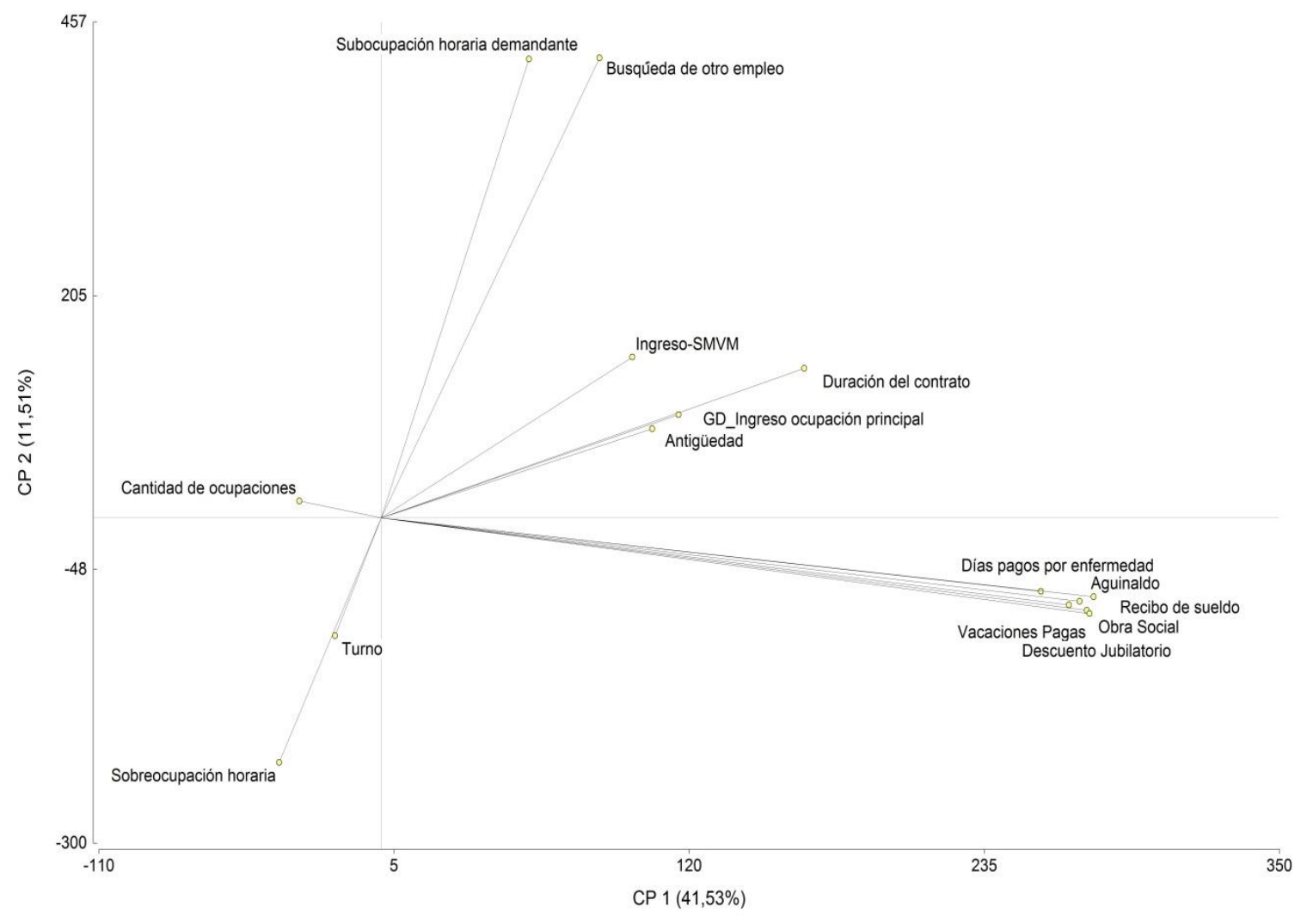

Fuente: elaboración propia sobre la base de datos de EPH-INDEC.

En la figura $N^{\circ} 1$ puede observarse un grupo de variables claramente diferenciadas en el eje de CP1, las denominadas "derechos laborales". Éstas son las que más variabilidad presentaron en esta componente (41,4\%). Se advertirse que asociadas a ellas, pero con mayor cercanía al eje de intersección, se encuentran "duración del contrato", "grupo decílico de ingreso de la ocupación principal", "antigüedad" e "ingreso-SMVM". Esta distribución puede ser de ayuda para profundizar el análisis y determinar si en aquellos casos donde existen trabajos que cumplan con los "derechos laborales" se asocian también contratos de duración indeterminada, salarios que superan el mínimo, vital y móvil y cuya estabilidad es mayor a un año. Para ello, sería pertinente aplicar un análisis de correspondencias múltiples.

El resto de las variables, muy cercanas al eje de intersección, no tienen importancia para explicar la variabilidad del CP1. Sí se observa para el eje CP2 (11,51\%) la importante distancia entre los casos que se agrupan con las variables "subocupación horaria" y "búsqueda de otro empleo" respecto de "sobreocupación". En ambos casos, se estima que la subocupación horaria demandante está relacionada con la búsqueda de otro empleo, situación que contrasta para los casos de sobreocupación horaria. 


\section{Conclusiones}

Tal como se observa en el análisis del aglomerado en cuestión, uno de los principales atributos que terminan por definir una situación de precariedad se relaciona con los aspectos regulatorios del trabajo. Criterios fundamentales como registro, obra social, aguinaldo, vacaciones, comprobantes de pago, entre otros, suelen ser variables lo suficientemente confiables como para estimar a nivel general la presencia de una relación de precariedad. Aspectos como el salarial e intensidad laboral son otros de gran impacto que pueden enriquecer las explicaciones relacionadas a este fenómeno.

La precariedad laboral es un fenómeno complejo y heterogéneo con relación a sus expresiones concretas entre los/as trabajadores/as, esto no sólo es motivo de debate académico respecto de las variables de mayor relevancia, sino también sobre sus estrategias de abordaje. Se reconoce a la precariedad como un fenómeno multidimensional, difícil de medir, cuyas expresiones particulares pueden depender de la conformación del mercado de trabajo y la regulación -legislación- existente sobre el las relaciones de trabajo.

El ejercicio estadístico llevado a cabo en este trabajo señala las variables que pueden explicar con más pertinencia las diferentes expresiones de precariedad. La amplitud de situaciones en que los conjuntos factoriales pueden ser utilizados brinda gran versatilidad en tanto análisis sobre sectores de actividad económica, por ejemplo, como muestran los trabajos de Fernández Massi (2014) y Pol, Guilló y Maradona (2015). También permiten encontrar algunas relaciones latentes entre variables y puede ser un ejercicio interesante de ser utilizado antes de analizar en profundidad alguna expresión del fenómeno de precariedad, por ello además a este tipo de estudios resultaría pertinente aplicar otras técnicas de análisis multivariados, como análisis de correspondencias múltiples y análisis de conglomerados.

Anexo 1:

Cuadro $N^{\circ}$ 6: Medidas resumen y datos faltantes. Valores muestrales.

\begin{tabular}{|l|l|l|}
\hline Variable & n & Datos faltantes \\
\hline Cantidad de ocupaciones & 477 & 0 \\
\hline Búsqueda de otro empleo & 477 & 0 \\
\hline Subocupación horaria demandante & 477 & 0 \\
\hline Sobreocupación horaria & 477 & 0 \\
\hline Antigüedad & 476 & 1 \\
\hline Turno & 476 & 1 \\
\hline Recibo de sueldo & 476 & 1 \\
\hline Duración del contrato & 476 & 1 \\
\hline Vacaciones pagas & 476 & 1 \\
\hline Aguinaldo & 476 & 1 \\
\hline Días pagos por enfermedad & 476 & 1 \\
\hline
\end{tabular}




\begin{tabular}{|l|l|l|}
\hline Obra social & 476 & 1 \\
\hline Descuento jubilatorio & 477 & 0 \\
\hline Ingreso-SMVM & 466 & 11 \\
\hline GD_Ingreso ocupación principal & 466 & 11 \\
\hline
\end{tabular}

Fuente: elaboración propia sobre la base de la Encuesta Permanente de Hogares (EPH).

\section{Bibliografía}

Acosta Reveles, I. (2011). ¿Flexibilidad salarial y la precarización del empleo en la agricultura latinoamericana? En Espacio Abierto. Cuaderno Venezolano de Sociología, $204,621-640$.

Arango, L.; Escobar, D. y Monsalve, M. (2013). Subempleo por ingresos y funcionamiento del mercado de trabajo en Colombia. En Revista Desarrollo y Sociedad, 72, 157-203.

Antunes, R. (2003). ¿Adiós al Trabajo? Ensayo sobre metamorfosis del trabajo y el rol central del trabajo. Buenos Aires: Ed. Herramienta.

Antunes, R. (2005). Los sentidos del trabajo. Ensayo sobre la afirmación y la negación del trabajo. En Herramienta-TEL, Buenos Aires, Argentina.

Antunes, R. y Braga, R. (2009). Infoproletários: degradação real do trabalho virtual. Brasil: Boitempo editorial.

Altimir, O. y Beccaria, L. (1999). Distribución del ingreso en Argentina. En Serie Reformas Económicas, 40. Chile: CEPAL.

Barattini, M. (2009). El trabajo precario en la era de la globalización. ¿Es posible la organización? En Polis. Revista Latinoamericana, 24. Disponible en: https://polis.revues.org/1071

Berasueta, A y Biaffore, E (2010). Principales reformas normativas en el ámbito laboral. Período 2002/2009. En Neffa, J.; Panigo, D. y Pérez, P. (ed.) Transformaciones del empleo en la Argentina: estructura, dinámica e instituciones. Buenos Aires: CICCUSCEIL.

Bouffartigue, P. (2007). Précarités professionnelles et action collective. La forme syndicale à l'épreuve. Mimeo. LEST.

Battistini, O. (2001). Flexibilización laboral en Argentina. Un camino hacia la precarización y la desocupación. En Revista Venezolana de Gerencia, Maracaibo.

Castel, R. (2010). El acenso de las incertidumbres: trabajo, protecciones, estatuto del individuo. Buenos Aires, Argentina: FCE.

Castillo Fernández, D.; Baca Tavira, N. y Todaro Cavallero, R. (2016). Trabajo global y desigualdades en el mercado laboral, Universidad Autónoma del Estado de México, México. 
Caire, G. (1982). Precarisation des emplois et regulation du marche du travail. Sociologie du travail 2.

Del Bono, A. y Bulloni, M. (2013). Los claroscuros de la revitalización sindical en contextos de tercerización: un apunte regional sobre el sector de call centers en Argentina. En Senén González, C. y Del Bono, A. (ed.) La revitalización sindical en Argentina: alcances y perspectivas. Buenos Aires Argentina: Universidad Nacional de la Matanza, Editorial Prometeo.

De la Garza Toledo, E. (2009). Los estudios laborales en América Latina al inicio del siglo XXI. En Revista Ciencia UAQ, 2, 2, 3-24.

De la Garza, E. y Neffa, J. (2010). Modelos económicos, modelo productivo y estrategias de ganancia: conceptos y problematización. En De la Garza, E. y Julio C. Neffa (coords.) Trabajo y modelos productivos en América Latina: Argentina, Brasil, Colombia, México y Venezuela luego de las crisis del modo de desarrollo neoliberal. Buenos Aires: CLACSO, 15-47 pp.

De la Garza Toledo, E. (2011). Trabajo no clásico, organización y acción colectiva. Universidad Autónoma Metropolitana-Iztapalapa, México.

De la Garza Toledo, E. (2012). La situación del trabajo en México. Madrid, España: Plaza y Valdés Editores.

EPH-INDEC (2004). Encuesta Permanente de Hogares. Mercado de trabajo, principales indicadores. Resultados del segundo trimestre de 2003. Instituto Nacional de Estadísticas y Censos (INDEC), Ciudad Autónoma de Buenos Aires, Argentina.

EPH-INDEC (2005). Encuesta Permanente de Hogares. Indicadores Socio-económicos. Resultados del cuarto trimestre de 2004. Instituto Nacional de Estadísticas y Censos (INDEC), Ciudad Autónoma de Buenos Aires, Argentina.

EPH-INDEC (2015a). Encuesta Permanente de Hogares. Mercado de trabajo, principales indicadores. Resultados del segundo trimestre de 2015. Instituto Nacional de Estadísticas y Censos (INDEC), Ciudad Autónoma de Buenos Aires, Argentina.

EPH-INDEC (2015b). Encuesta Permanente de Hogares. Indicadores Socio-económicos. Resultados del cuarto trimestre de 2014. Instituto Nacional de Estadísticas y Censos (INDEC), Ciudad Autónoma de Buenos Aires, Argentina.

Fernández Massi, M. (2014). Dimensiones de la precariedad laboral: un mapa de las características del empleo sectorial en la Argentina. En Cuadernos de Economía, 33, 62, 231-257.

Fernández Milmanda, B. (2013). Cuellos no tan blancos. Los trabajadores de supermercados y su desafío al viejo modelo sindical mercantil. En Senén González, C. y Del Bono, A. (eds.) La revitalización sindical en Argentina: alcances y perspectivas. Buenos Aires, Argentina: Universidad Nacional de la Matanza, Editorial Prometeo.

Feldman, S. y Galin, P. (1990). Nota introductoria. En Galin, P. y Novick, M. (comp.) La precarización del empleo en la Argentina. Buenos Aires: CEAL. 
García, B. y De Oliveira, O. (2012). Trabajo e ingresos de los miembros de las familias en el México metropolitano. En De la Garza Toledo, E. (comp.) La situación del trabajo en México. Madrid, España: Plaza y Valdés Editores.

García Guzmán, B. (2008). Los mercados de trabajo urbanos de México a principios del siglo XXI. En Revista Mexicana de Sociología, 71, 46-105.

Gorz, A. (1995). Metamorfosis del trabajo. Búsqueda del sentido. Crítica de la razón económica. Madrid, España: Editorial Sistema.

Giosa Zuazúa, N. (2007). Transformaciones y empleo en el mercado de empleo en la Argentina. Entre el desempleo y el empleo precario. En Basualdo, V.; Forcintito, K.; Rodríguez, J.; Seain, C. y Sztulwark, S. (comp.) Transformaciones recientes en la Economía Argentina. Buenos Aires: UNGS, Prometeo.

Guadarrama Olivera, R.; Hualde Alfaro, A. y López Estrada, S. (2012). Precariedad laboral y heterogeneidad ocupacional: una propuesta teórico-metodológica. En Revista Mexicana de Sociología 2, 74, 213-243.

Henry, L. (2009). Flexibilización de los vínculos laborales y formas de inserción precarias en la prensa escrita. En $9^{\circ}$ Congreso Nacional de Estudios del Trabajo. Buenos Aires, 5-7 de agosto.

Henry, L. (2013). Fragmentación y precarización laboral en la prensa escrita. Los desafíos para la representación y la organización colectiva de los periodistas en un entorno productivo flexibilizado. En Senén González, C. y Del Bono, A. (eds.) La revitalización sindical en Argentina: alcances y perspectivas. Buenos Aires, Argentina: Universidad Nacional de la Matanza, Editorial Prometeo.

Julian Vejar, D. (2013). Trabajo, precariedad y "habitus precario". Aproximaciones al estudio de la(s) precariedad(es) en América Latina. En Revista Latinoamericana de Estudos do Trabalho 30, 18, 185-210.

Mora Salas, M. (2009). Ajuste y empleo: tendencias de precarización del trabajo asalariado. México: El Colegio de México.

Merklen, D. (2004). Sobre la base territorial de la movilización popular y sobre sus huellas en la acción. En Lavboratorio. Revista de Estudios sobre Cambio Social, 4, 6.

Medina, F. y Galván, M. (2007). Imputación de datos: teoría y práctica. Serie Estudios estadísticos y prospectivos. División de Estadística y Proyecciones Económicas, CEPAL, Santiago de Chile, Chile.

MTEySS y OIT (2012). Macroeconomía, empleo e ingresos: debates y políticas en Argentina frente a la crisis internacional 2008-2009. Buenos Aires: Oficina de País de la OIT para la Argentina.

Muñoz Izquierdo, C. (2006). Determinantes de la empleabilidad de los jóvenes universitarios y alternativas para promoverla. En Papeles de Población 12, 49, 75-89.

Neffa, J. C (Coord.); Alvarez Hayes, S; Battistuzzi, A; Biaffrone, E y Suárez Maestre, A (2008). La informalidad, la precariedad laboral y el empleo no registrado en la 
provincia de Buenos Aires. CEIL-PIETTE CONICET. Buenos Aires, Argentina: Ed CLACSO.

Neffa, J. C. (2010). Modelos productivos y sus impactos sobre la relación salarial. Reflexiones a partir del caso Argentino. En Neffa, J. y De la Garza Toledo, E. (eds.) Trabajo y modelos productivos en Latinoamérica: Argentina, Brasil, Colombia, México y Venezuela luego de las crisis del modo de desarrollo neoliberal. Buenos Aires, Argentina: Ed. Consejo Latinoamericano de Ciencias Sociales (CLACSO).

Organización Internacional del Trabajo (OIT) (2003). Las consecuencias para el empleo de las fusiones y adquisiciones en el sector del comercio, Ginebra, Suiza.

Organización Internacional del Trabajo (OIT) (2012). Del trabajo precario al trabajo decente: documento final del simposio de los trabajadores sobre políticas y reglamentación para luchar contra el empleo precario. Ginebra, Suiza: Oficina Internacional del Trabajo, Oficina de Actividades para los Trabajadores (ACTTRAV).

Organización Internacional del Trabajo (OIT) (2015). Perspectivas sociales y del empleo en el mundo. El empleo en plena mutación. Resumen ejecutivo.

Osorio, J. (2013). Sobre dialéctica, superexplotación y dependencia. Notas acerca de Dialéctica de la dependencia. En Revista Argumentos, 72, 26, 57-73.

Pok, C. (1992). Precariedad laboral: personificaciones sociales en la frontera de la estructura de empleo. Documento de trabajo 29. Buenos Aires, Argentina: CEILPIETTE.

Pol, M.; Guilló, M. y Maradona, G. (2015). Precariedad laboral en el Gran Mendoza: un análisis de sus dimensiones. En VIII Pre-ASET, Mendoza, 14-15 de mayo.

Pacheco, E.; De la Garza, E. y Reygadas, L. (2010). Trabajos atípicos y precarización del empleo. México: Colegio de México.

Rodgers, G. (1992). El debate sobre el trabajo precario en Europa Occidental. En Rodgers, G. y Rodgers, P. (comps.) El trabajo precario en la regulación del mercado laboral: crecimiento del empleo atípico en Europa Occidental. Madrid.

Salvia, A. (2002). Segmentación de la estructura social del trabajo. El problema, debate y alternativas. En Lavboratorio: Informe de Coyuntura Laboral, 4, 9.

Senén González, C. y Haidar, J. (2009). Los debates acerca de la "revitalización sindical" y su aplicación en el análisis sectorial en Argentina. En Revista Latinoamericana de Estudios del Trabajo (RELET), 22, 14, 5-32. 